\title{
Model Based Control of Wind Turbines: Look-Ahead Approach
}

\author{
Alexander Stotsky* and Bo Egardt ${ }^{* *}$ \\ * Signals \& Systems, Chalmers University of Technology, Gothenburg, \\ SE-41296, Sweden (e-mail: alexander.stotsky@chalmers.se) \\ ** Signals \& Systems, Chalmers University of Technology, Gothenburg, \\ SE-41296, Sweden (e-mail: bo.egardt@chalmers.se)
}

\begin{abstract}
A new composite turbine control architecture that consists of feedforward and feedback parts based on the upwind speed measurements and wind speed measurements at the turbine site, respectively, is described. The algorithm starts with preprocessing of a low rate sampled upwind speed via spline interpolation method. A run-ahead model driven by the signals from a preprocessing block models the turbine response and produces the feedforward part of turbine controller. The turbine control system is driven by both feedforward part which comes from the run-ahead model, and feedback part based on the wind speed measured at the turbine site. It is proved that the controller is stable despite the difference between the time shifted preview measurements (expected wind speed) and actual wind speed measured at the turbine site. Existing industrial PI/PID turbine controllers can easily be upgraded with the preview part of the control architecture described in this paper. Improved blade load regulation via the blade pitch angle control guarantees a hard upper bound on the flapwise bending moment. The results are confirmed by simulation with a wind speed record from the Hönö turbine outside Gothenburg, Sweden.
\end{abstract}

Keywords: Wind turbine, Preview control, Look-ahead modeling, Feedforward, Spline interpolation, Load mitigation, Wind turbine model reduction

\section{INTRODUCTION}

The uncontrollable stochastic nature of the wind necessitates prediction of wind speed to achieve high performance turbine regulation. Two control architectures that use the preview of wind speed are known. The first one is based on wind speed measurements at the turbine site and prediction of the future changes of wind speed using a time-series model (see for example [1] for a one step ahead prediction). The second one (see for example [2]-[9] and references therein) requires equipment for measuring wind speed at a distance in front of the turbine. New laser sensor technologies are the most promising techniques of measuring upwind speed which open new opportunities for development of forward-looking turbine control strategies with their subsequent integration into existing turbine control system.

The first strategy suffers from modeling/prediction errors, and the advantages of the second one might be diminished by the difference between upwind speed and the speed that arrives to the turbine site.

The second prediction scheme gives more opportunities for performance improvement, but requires installation of the additional measurement equipment, which might be expensive. Preview based control strategies are usually based on an assumption that the same wind speed which is measured at a distance in front of the turbine comes to the turbine. This assumption is often not valid in practice. Moreover, a classical frozen turbulence assumption used for calculation of expected wind speed at the turbine site introduces additional inaccuracies in preview information. Besides, the laser preview measurements of the wind speed are usually provided with a relatively low sampling rate [9]. As a rule the laser update rates do not exceed ten $H z$, and the most common rate is one $H z$, although higher sample rates will be available at low cost in the future. Measurement rates of other system variables such as generator speed are much higher. Such a low sampling rate applied to a turbulent wind results in filtering of a fast varying wind speed due to aliasing effect, where high frequencies are aliased to a low frequency range. Such an effect is accompanied by errors/inaccuracies in preview measurements and increase the difference between upwind speed measurements and measurements of the speed that arrives at the turbine site. This in turn necessitates the development of a new control architecture driven by both upwind speed and wind speed measurements at the turbine site (provided by the cup anemometer installed on the nacelle or by laser that measures wind speed at a short distance in front of the turbine) for improvement of the control accuracy.

Wind speed signal measured at a distance in front of the turbine allows preprocessing and generation of high quality feedforward control signal. A feedback control loop is usually based on wind speed measurements at the turbine site. An integration of the feedforward part driven by upwind speed and feedback part driven by wind speed at the turbine site into a control system is an issue since those parts might result in conflicting control actions. This paper offers the design method for a new control architecture 

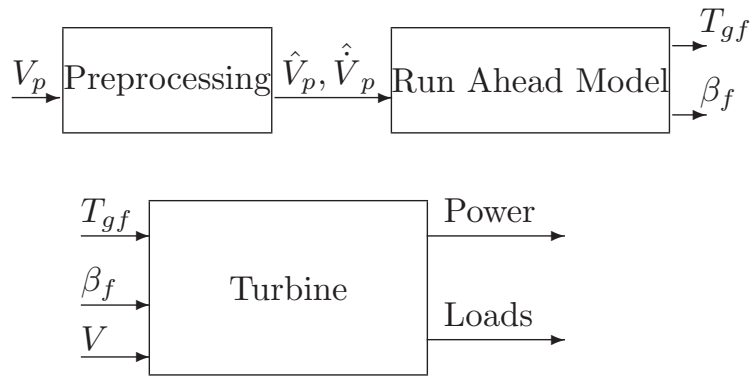

FIG 1. Block diagram that describes the structure of the turbine control system with run-ahead model in the loop.

that successfully integrates those feedback and feedforward parts.

The main contribution of this paper is a new control architecture that integrates: 1) a signal processing block for preprocessing of the wind speed signal; 2) a run-ahead model that models the turbine response driven by the preview measurements and exports the feedforward part to the turbine controller; 3) a feedback part driven by the wind speed measurements at the turbine site.

Blade load mitigation is achieved via enhanced pitch angle regulation.

A block diagram of the proposed control structure is shown in Figure 1, where upwind speed $V_{p}$, measured at a distance in front of the turbine is an input to preprocessing block which cleans the signal and calculates a high quality wind speed signal $\hat{V}_{p}$ and its derivative $\hat{\dot{V}}_{p}$ with some time delay. A run-ahead model driven by the signals from a preprocessing block models the turbine response and produces feedforward parts of the controller: generator torque $T_{g f}$, and blade pitch angle $\beta_{f}$. Turbine control system is driven by both feedforward part which comes from run-ahead model and feedback part based on wind speed $V$ measured at the turbine site.

A satisfactory performance of the closed loop system despite a mismatch between the speed expected and measured at the turbine site is shown using measured wind speed data and simple turbine model. Moreover, the stability of the closed loop system is proved for the case of constant mismatch between those speeds.

The paper is organized as follows. Turbine model and problem statement are described in Section 2. Composite controller which contains a preview based part presented in Section 3 is described and verified by simulations in Section 4. The paper is finished with brief conclusions in Section 5 .

\section{TURBINE MODEL}

The description of the turbine model begins with an aerodynamical part, and drive train and pitch actuator models. Steady-state blade operational loads and the trajectory tracking problem statement are carried over from [8]. The model is completed by wind speed measurements made in front of $H \ddot{o} n \ddot{o}$ turbine.

\subsection{Aerodynamic Model}

A wind turbine converts energy from the wind to the rotor shaft that rotates at a speed $\omega_{r}$. The power of the wind $P_{\text {wind }}=\frac{1}{2} \rho A V^{3}$ depends on the wind speed $V$, the air density $\rho$, and the swept area $A=\pi R^{2}$, where $R$ is the rotor radius. Uniform distribution of the wind speed across the rotor swept area is assumed. From the available power in the swept area, the power on the rotor $P_{r}$ is given based on the power coefficient $C_{p}(\lambda, \beta)=\frac{P_{r}}{P_{\text {wind }}}$ which in turn, depends on the pitch angle of the blades $\beta$ and the tipspeed ratio $\lambda=\frac{\omega_{r} R}{V}$ :

$$
P_{r}=P_{\text {wind }} C_{p}(\lambda, \beta)=\frac{A \rho V^{3} C_{p}(\lambda, \beta)}{2}
$$

The aerodynamic torque applied to the rotor is given as:

$$
T_{a}=\frac{P_{r}}{\omega_{r}}=\frac{A \rho V^{3} C_{p}(\lambda, \beta)}{2 \omega_{r}}
$$

\subsection{Reduced-Order Modeling of the Drive Train}

The drive train model consists of a low-speed shaft rotating with a speed $\omega_{r}$ and a high-speed shaft rotating with a speed $\omega_{g}$, having inertias $J_{r}$ and $J_{g}$ respectively. The shafts are interconnected by a gear with ratio $N$. A torsion stiffness $K_{s}$ together with a torsion damping $K_{d}$ result in a torsion angle $\alpha$ that describes the twist of the flexible shaft. This leads to the following drive train model [10]:

$$
\begin{aligned}
J_{r} \dot{\omega}_{r} & =\underbrace{\frac{P_{r}}{\omega_{r}}}_{=T_{a}}-\underbrace{K_{s} \alpha-K_{d} \dot{\alpha}}_{\text {torque shared by the shafts }} \\
J_{g} \dot{\omega}_{g} & =\frac{K_{s}}{N} \alpha+\frac{K_{d}}{N} \dot{\alpha}-T_{g} \\
\dot{\alpha} & =\omega_{r}-\frac{1}{N} \omega_{g}
\end{aligned}
$$

Multiplication of both sides of (4) by $N$ and subsequent summation with (3) yields:

$$
J_{r} \dot{\omega}_{r}+N J_{g} \dot{\omega}_{g}=\frac{P_{r}}{\omega_{r}}-N T_{g}
$$

where the term $K_{s} \alpha+K_{d} \dot{\alpha}$ which represents the torque between the shafts is canceled. The torsion rate $\dot{\alpha}$ is several dozen times smaller than the turbine speed $\omega_{r}$. Therefore, a generator speed divided by a gear ratio is an acceptable approximation of the turbine speed, $0=\omega_{r}-\frac{1}{N} \omega_{g}$. Finally, a one mass nonlinear model of the drive train with the loading torque from generator $T_{g}$ as a control action, with a turbine power $P_{r}$ as an input, and with a rotational speed $\omega_{r}$ as an output can be presented as follows:

$$
\begin{gathered}
\omega_{g}=N \omega_{r} \\
J \dot{\omega}_{r}=\underbrace{\frac{P_{r}}{N \omega_{r}}}-T_{g} \\
=\frac{T_{a}}{N}
\end{gathered}
$$

where $J=\frac{J_{r}+N^{2} J_{g}}{N}$ is a lumped rotational inertia of the system.

A nomenclature and parameters of the turbine model described above are presented in [8]. 


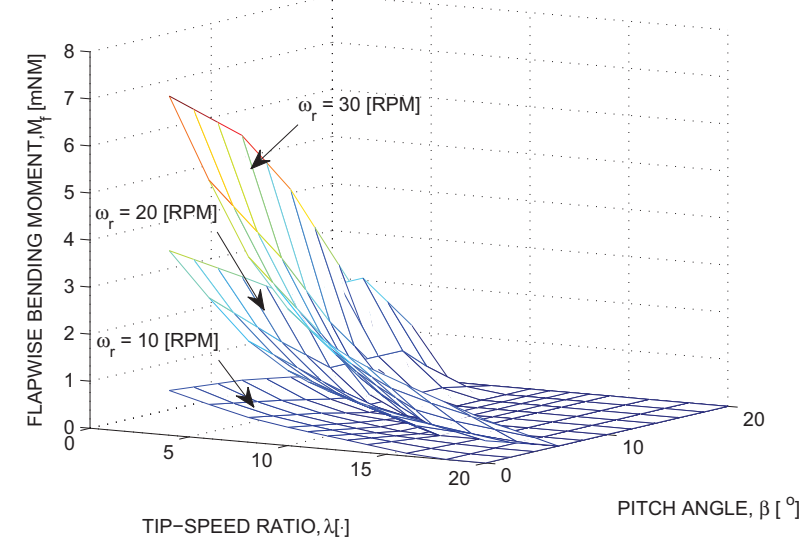

FIG 2. Flapwise bending moment as a function of tip-speed ratio and pitch angle for different values of turbine speed.

\subsection{Pitch Actuator Model}

Pitch actuator is modeled as a first order lag with the rate and range constraints:

$$
\begin{aligned}
\dot{\beta} & =-\frac{1}{\tau} \beta+\frac{1}{\tau} \beta_{d}\left(t-t_{d}\right) \\
|\beta| & \leq C_{\beta}, \quad|\dot{\beta}| \leq C_{\dot{\beta}}
\end{aligned}
$$

where $\beta_{d}\left(t-t_{d}\right)$ is an actuator control input, $\tau$ is a time constant, $t_{d}$ is a communication delay, and $C_{\beta}$ and $C_{\dot{\beta}}$ are positive constants which define range and rate constraints respectively.

\subsection{Steady-State Blade Operational Loads}

The steady-state flapwise and edgewise blade root bending moments can be described as look-up tables (surfaces in three dimensional space) with tip-speed ratio and blade pitch angle as input variables [8],[11]. Such sandwiched surface that describes flapwise blade bending moment as a function of tip-speed ratio and blade pitch angle for different turbine speeds is plotted in Figure 2.

\subsection{Wind Speed Measurements}

Wind speed measurements made on Hönö wind turbine outside Gothenburg, Sweden [12] with sampling rate of $1 \mathrm{~Hz}$ are directly used in simulations. The wind speed measurement setup is shown in Figure 3, and measured data are plotted in Figure 4.

\subsection{A Turbine Control Problem Statement}

Turbine control problem is to choose a desired generator torque $T_{g}$ and pitch actuator input $\beta_{d}$ in order to maximize a turbine power $P_{r}$ under the constraints on the flapwise and edgewise bending moments:

$$
\begin{array}{r}
P_{r} \rightarrow P_{r \max } \\
M_{f}\left(V, \omega_{r}, \beta\right) \leq C_{f} \\
M_{e}\left(V, \omega_{r}, \beta\right) \leq C_{e}
\end{array}
$$

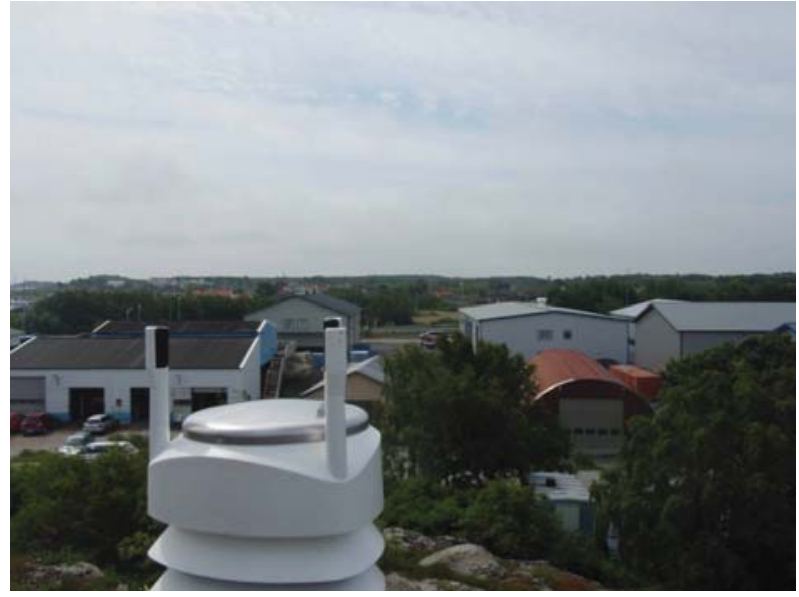

FIG 3. Wind speed measurements with WXT520 Vaisala wind speed sensor located in front of Hönö wind turbine outside Gothenburg, Sweden. The sensor has an array of three equally spaced ultrasonic transducers on a horizontal plane. A wind speed and direction are determined by measuring the time it takes the ultrasound to travel from each transducer to the other two.

where $P_{\text {rmax }}$ is the maximum turbine power available under the constraints (12), (13), and $C_{f}$ and $C_{e}$ are positive constants. Moments $M_{f}(\cdot)$ and $M_{e}(\cdot)$ are steadystate flapwise and edgewise bending moments respectively. This problem statement, described in [8] can be converted to a driveline control problem i.e., to choose a desired generator torque $T_{g}$ and pitch actuator input $\beta_{d}$ to track a desired turbine speed $\omega_{r d}$, and blade pitch angle $\beta_{f}$ :

$$
\begin{array}{r}
\lim _{t \rightarrow \infty} \omega_{r}(t)-\omega_{r d}=0 \\
\lim _{t \rightarrow \infty} \beta(t)-\beta_{f}=0
\end{array}
$$

where $\omega_{r d}$ and $\beta_{f}$ are chosen to maximize the power coefficient $C_{p}(\lambda, \beta)$ in the presence of constraints on the blade bending moments.

This problem statement does not describe the typical division of wind turbine control into operating regions, where wind speed is below/above rated speed [13] and provides a unified description for both regions. The case where wind speed is above rated is accounted via constraints on blade bending moments (12),(13). Indeed, the wind speed above rated implies high loads on the blades which exceed desired limits. Pitch actuation, similar to [13] is applied in this case to keep the loads under constraints. Constraints on blade loads are not violated, if the wind speed is below rated. Pitch actuation is not applied in this case, maximizing power output of the turbine.

Moreover, even if the wind speed is below rated an additional pitch actuation might be introduced within this problem statement framework via tougher constraints on blade loads. Additional bounding of blade loads might be required a) in the case of ageing and wearing of the turbine components, b) for offshore turbines in the case of nasty sea states, c) in the case of surface roughness on the blades, arising from turbine icing in cold climate, and d) in many other cases [8]. 


\section{PREVIEW BASED CONTROL}

The description of a preview based control strategy starts with the spline interpolation preprocessing of wind speed signal, and look ahead modeling of the turbine response. A preview based control results in feedforward part of the turbine controller.

\subsection{Preprocessing of Wind Speed Signal}

Wind speed signal $V_{p}$ measured at a distance in front of the turbine with a relatively low sampling rate (compared to other signals of the system) should properly be processed to achieve high performance regulation. Preprocessing of the wind speed signal includes estimation of the derivative of the signal for further inclusion in the control system. The backward difference method, which is well-known as the simplest numerical differentiator gives the derivative that is accompanied with peaking phenomena due to a low sampling rate of the signal. Spline interpolation method (see [14] and references therein) which is based on on-line least-squares polynomial fitting over the moving in time window of a size $w$ is proposed in this paper for calculation of the derivative of the wind speed signal. The idea for the spline interpolation method is to fit a polynomial of a certain order as a function of time to the measured upwind speed signal $V_{p}$ :

$$
\hat{V}_{p}=c_{0}+c_{1} t+\ldots+c_{n} t^{n}
$$

where $\hat{V}_{p}$ is an estimate of the signal, $t$ is a continuous time, $c_{i}, i=0, \ldots, n$ are the coefficients to be found, in a least squares sense and take the derivatives analytically. The sum to be minimized at every step is the following

$$
S_{k}=\sum_{j=k-(w-1)}^{j=k}\left(V_{p j}-\left(c_{0}+c_{1} t_{j}+\ldots+c_{n} t_{j}^{n}\right)\right)^{2}
$$

where $k=w, w+1, \ldots$

Recursive and computationally efficient version of the spline interpolation method is described in [14].

A preview based measurement strategy allows preprocessing of the wind speed signal and the derivatives of the signal can be taken in the middle of the moving window. This essentially improves an estimation accuracy and hence the performance of control system. Application of the spline interpolation method with the second order spline is illustrated in Figure 4, where a high performance derivative signal is created from the upwind speed signal with a low rate sampling. The wind speed signal in $[\mathrm{m} / \mathrm{sec}]$ is measured with the frequency of $1 \mathrm{~Hz}$ and plotted with a blue line, where constant offset of $5[\mathrm{~m} / \mathrm{sec}]$ is subtracted at each step. Second order polynomial (plotted with a black line) as a function of time is fitted to the measured signal in the least-squares sense in a window which is moving in time. The derivative (plotted with a red line) is calculated in the middle of this window.

Notice that spline interpolation method provides also higher order derivatives.

\subsection{Look-Ahead Modeling of the Turbine Response}

Upwind speed measurements allow look-ahead modeling (pre-modeling) of the turbine response and generation of high quality (almost noise-free) feedforward control signal.

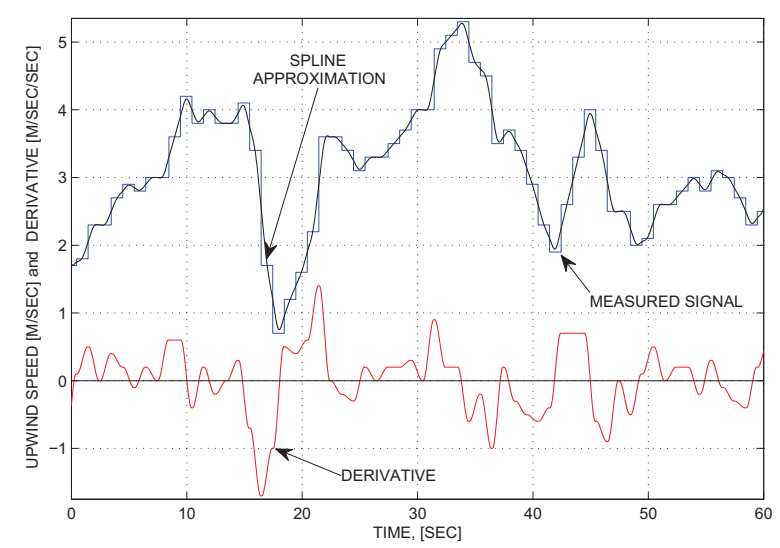

FIG 4. Numerical differentiation of the upwind speed signal via spline interpolation method.

Run-Ahead Model. Run-ahead turbine model is introduced as follows:

$$
\begin{aligned}
\hat{J} \dot{\omega}_{r m} & =\frac{P_{r m}}{N \omega_{r m}}-T_{g m} \\
P_{r m} & =\frac{A \rho V_{p}^{3} C_{p}\left(\lambda_{m}, \beta_{m}\right)}{2} \\
\lambda_{m} & =\frac{\omega_{r m} R}{V_{p}} \\
\dot{\beta}_{m} & =-\frac{1}{\tau} \beta_{m}+\frac{1}{\tau} \beta_{m d}\left(t-t_{d}\right)
\end{aligned}
$$

that models a virtual turbine located at a distance in front of real turbine with the wind speed $V_{p}$, turbine power $P_{r m}$, turbine speed $\omega_{r m}$, tip-speed ratio $\lambda_{m}$, and desired and actual blade pitch angles $\beta_{m d}$ and $\beta_{m}$ with rate and range constraints on $T_{g m}$ and $\beta_{m}$. The model is driven by a generator torque $T_{g m}$ to achieve the desired closed loop performance of the virtual turbine. To this end the desired values of tip-speed ratio $\lambda_{*}$ and blade pitch angle $\beta_{*}$ are calculated first using the approach described in [8]. It is assumed that the rotational inertia of the system $J$ is unknown due to a turbine icing in cold climate, for example. Constant a-priory value of inertia $\hat{J}$ is used in model (18).

Torque Control. The generator torque

$$
\begin{aligned}
T_{g m} & =\underbrace{\frac{P_{r m}}{N \omega_{r m d}}}_{\text {feedforward part }}+\underbrace{\gamma\left(\omega_{r m}-\omega_{r m d}\right)}_{\text {feedback part }} \\
& -\underbrace{\hat{J} \dot{\omega}_{r m d}}_{\text {predictive part }}
\end{aligned}
$$

where $\gamma>0$ guarantees a satisfactory closed loop tracking performance of desired speed of virtual turbine $\omega_{r m d}=$ $\frac{\lambda_{*} V_{p}}{R}$, where $\dot{\omega}_{r m d}=\frac{\lambda_{*} \dot{V}_{p}}{R}$ is calculated via a spline interpolation method as it is described in Section 3.1. This generator torque control consists of three parts: feedback and feedforward parts as well as a derivative driven part for accounting of fast changes of wind speed. 
Main idea of this controller becomes clear when substituting (22) in (18). This results in the following exponentially stable closed loop dynamics of virtual turbine:

$$
\hat{J}\left(\dot{\omega}_{r m}-\dot{\omega}_{r m d}\right)=-\left[\frac{P_{r m}}{N \omega_{r m} \omega_{r m d}}+\gamma\right]\left(\omega_{r m}-\omega_{r m d}\right)
$$

Notice that the generator torque can be made adaptive with an adjustable parameter multiplied by the derivative of desired turbine speed $\dot{\omega}_{r m d}$. Such an adaptation law compensates for estimation errors in the derivative of upwind speed. This case is not considered in the paper for the sake of simplicity.

Regulation of Flapwise Bending Moment. Mechanical loads of virtual turbine are regulated via pitch angle $\beta_{m}$. The controller for pitch angle is based on look-up tables that are inverse to the flapwise bending moment look-up tables $M_{f}\left(\lambda_{m}, \beta_{m}\right)$, shown in Figure 2. Those inverse lookup tables $M_{f}^{-1}\left(\lambda, M_{f d}\right)$ have two inputs: tip-speed ratio $\lambda_{m}$ and a desired flapwise bending moment $M_{f d}$. Pitch regulator is defined as $\beta_{m d}(t)=M_{f}^{-1}\left(\lambda_{m}, M_{f d}\right)$ for turbine speed $\omega_{r m}$, regulated by the control algorithm (22). As a desired value of flapwise bending moment $M_{f d}$, its desired upper bound can be taken. In other words the flapwise bending moment is directly regulated to keep this moment bounded.

Exporting Feedforward Signals. Cleaned and sampled with a high frequency the generator control signal $T_{g m}(t)$, calculated via (22) guarantees high performance regulation of the speed of virtual turbine to the desired one, which is driven by the upwind speed measurements. The high performance of the regulation is achieved due to the possibility of preprocessing of the wind speed signal, where a low-rate sampled signal is saved in the buffer, approximated via a polynomial as a function of time with continuous calculation of the derivative with the delayed time instant. The generator torque signal $T_{g m}(t)$ that guarantees the closed loop performance of virtual turbine is delayed and exported as a feedforward part to the controller for real turbine. Notice that any other runahead model might be used instead of model (18) - (21). However, low-order-look-ahead-modeling of the turbine response that captures low frequency component of the response is preferable. That in turn minimizes the errors due to a possible mismatch between the upwind speed and the speed that comes to the turbine.

Blade pitch angle $\beta_{m}(t)$ as function of time taken from run-ahead model (21) can also be exported as feedforward part to the blade pitch regulator of real turbine. A slowly varying (measured with $1 \mathrm{~Hz}$ sampling frequency) upwind speed profile allows high performance tracking of desired speed of virtual turbine and regulation of tip-speed ratio to an optimal value $\lambda_{*}$. This in turn results in small variations of pitch angle $\beta_{m}$, regulated via the algorithm described in Section 3.2.3, around desired value $\beta_{*}$. Therefore desired blade pitch angle is exported as feedforward part to the controller for real turbine.

\section{COMPOSITE TURBINE SPEED CONTROL: INTEGRATION OF FEEDFORWARD AND FEEDBACK PARTS}

This Section starts with an integration of feedback and feedforward parts into a composite turbine speed control architecture, where the feedforward part is imported from Section 3. Simulation results of the composite turbine speed control with improved blade load regulation are presented in the end of this Section.

\subsection{Turbine Speed Control}

Wind speed measured at a distance in front of the turbine and delayed for processing $V_{p}(t-\tau)$ with the derivative $\dot{V}_{p}(t-\tau)$ comes to the turbine at a time $t$ and denoted as $V_{p}(t)$ with the derivative $\dot{V}_{p}(t)$, where $\tau>0$ is elapsed time required for an upwind speed profile to reach the turbine. Wind speed measured at the turbine site $V(t)$ is composed of two components: the first one is measured upwind speed that reached the turbine $V_{p}(t)$ (expected wind speed) and the second one represents the deviation of expected speed from actual one $\Delta V(t)$ i.e., $V(t)=V_{p}(t)+\Delta V(t)$. This presentation of the turbine wind speed justifies the composite structure of controller that is composed of two components: the first component is a feedforward one driven by the upwind speed $V_{p}(t)$ and run-ahead model $T_{g m}(t-\tau)=T_{g f}(t)$, and the second one is feedback component driven by the wind speed mismatch $\Delta V(t)$.

Assuming that the wind speed mismatch $\Delta V$ is constant, the desired turbine speed and its derivative are defined as follows:

$$
\begin{aligned}
& \omega_{r d}=\frac{\lambda_{*} V}{R}=\underbrace{\frac{\lambda_{*} V_{p}}{R}+\underbrace{\frac{\lambda_{*} \Delta V}{R}}_{=\text {const }}}_{=\omega_{r m d}} \\
& \dot{\omega}_{r d}=\dot{\omega}_{r m d}=\frac{\lambda_{*} \dot{V}_{p}}{R}
\end{aligned}
$$

which show that the derivative of the desired turbine speed coincides with the derivative of the desired turbine speed of run-ahead model for a constant $\lambda_{*}$.

The simplest control strategy (similar to (22)) that uses a calculated ahead derivative of the wind speed signal can be written as follows:

$$
T_{g}=\frac{P_{r}}{N \omega_{r d}}+\gamma_{r}\left(\omega_{r}-\omega_{r d}\right)-J \dot{\omega}_{r d}
$$

where $\omega_{r d}$ and $\dot{\omega}_{r d}$ are defined in (24) and (25) respectively, with the derivative $\dot{V}_{p}$, cleaned from the noise in the preprocessing, $\gamma_{r}>0$, when assuming that the inertia of the system $J$ is known. This strategy, when combining (8) with (26), results in the following exponentially stable closed loop dynamics:

$$
J\left(\dot{\omega}_{r}-\dot{\omega}_{r d}\right)=-\left[\frac{P_{r}}{N \omega_{r} \omega_{r d}}+\gamma_{r}\right]\left(\omega_{r}-\omega_{r d}\right)
$$

despite a constant mismatch between $V$ and $V_{p}$.

However, physical look-ahead modeling of the wind turbine response gives more sophisticated control strategy based on high quality signal of the generator torque $T_{g m}(t)$ 

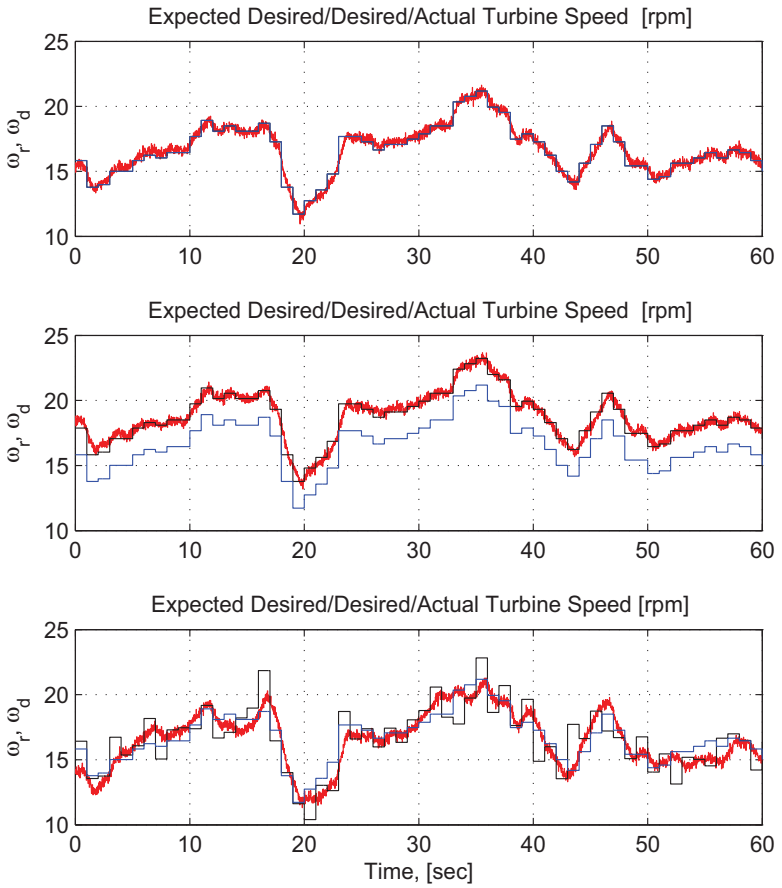

FIG 5. The time chart of turbine speed tracking performance for different mismatch $\Delta V$ between wind speed measured at a distance in front of the turbine $V_{p}$ (and expected at the turbine site after some time) and wind speed measured at the turbine site $V=V_{p}+\Delta V$.

sampled with a high frequency that guarantees high performance regulation driven by the upwind speed measurements only. The generator torque $T_{g m}(t)$, obtained from look-ahead modeling of the virtual turbine response and imported as feedforward part of the controller can be seen as a control that is tolerant to the wind/turbine speed sensor faults since this torque depends on the upwind speed measurements only.

A composite generator torque control strategy can be presented in the following form:

$$
T_{g}=\underbrace{\underbrace{T_{g m}}_{\text {feedforward part }}+\underbrace{\gamma_{r}\left(\omega_{r}-\omega_{r d}\right)+\gamma_{r 1} \int\left(\omega_{r}-\omega_{r d}\right)}_{\text {feedback part }}(28)}_{\text {composite controller }}
$$

where feedforward torque $T_{g m}$ is given by (22) and driven by the upwind measurements. Proportional-integral, PI feedback part with positive coefficients $\gamma_{r}$, and $\gamma_{r 1}$ is driven by the wind speed measured at the turbine site. A closed loop dynamics is examined via a substitution of (22) in (28) and (8) :

$$
\begin{aligned}
J \dot{\omega}_{r}= & \frac{P_{r}}{N \omega_{r}}-\underbrace{\frac{P_{r m}}{N \omega_{r m d}}-\gamma \underbrace{\left(\omega_{r m}-\omega_{r m d}\right)}_{\rightarrow 0}+\hat{J} \underbrace{\dot{\omega}_{r m d}}_{=\dot{\omega}_{r d}}}_{=-T_{g m} \text { (imported feedforward part) }} \\
& \underbrace{-\gamma_{r}\left(\omega_{r}-\omega_{r d}\right)-\gamma_{r 1} \int\left(\omega_{r}-\omega_{r d}\right)}_{\text {feedback part }}
\end{aligned}
$$

The term $\left(\omega_{r m}-\omega_{r m d}\right)$ is vanishing, since the speed of run-ahead model $\omega_{r m}$ converges to desired speed $\omega_{r m d}$ and can be neglected for the sake of simplicity. The difference between actual and modeled turbine torques and inertia moment can be presented as follows: $\frac{P_{r}}{N \omega_{r}}-$ $\frac{P_{r}}{N \omega_{r d}}+\frac{P_{r}}{N \omega_{r d}}-\frac{P_{r m}}{N \omega_{r m d}}$, and $\hat{J}=J+\Delta J$. It can be shown that the difference $\frac{P_{r}}{N \omega_{r d}}-\frac{P_{r m}}{N \omega_{r m d}}$ is approximately constant around an operating point. The term $\Delta J \dot{\omega}_{r d}$ can be treated as a constant around an operating point, assuming a piecewise linear wind speed. Therefore (29) can be written as follows:

$$
\begin{gathered}
J \dot{\omega}_{r}=\frac{P_{r}}{N \omega_{r}}-\frac{P_{r}}{N \omega_{r d}}+J \dot{\omega}_{r d}-\gamma_{r}\left(\omega_{r}-\omega_{r d}\right) \\
-\gamma_{r 1} \int\left(\omega_{r}-\omega_{r d}\right)+c
\end{gathered}
$$

where $c$ is a lumped constant. Error model (30) can be rewritten in the following form:

$$
\begin{aligned}
& \dot{\tilde{\omega}}_{r 1}=\tilde{\omega}_{r} \\
& J \dot{\tilde{\omega}}_{r}=-\left[\frac{P_{r}}{N \omega_{r} \omega_{r d}}+\gamma_{r}\right] \tilde{\omega}_{r}-\gamma_{r 1} \tilde{\omega}_{r 1}+c
\end{aligned}
$$

where $\tilde{\omega}_{r}=\omega_{r}-\omega_{r d}$. This model represents a stable dynamics with a performance regulated by the coefficients $\gamma_{r}$ and $\gamma_{r 1}$. Therefore the turbine speed converges to the desired speed with guaranteed performance.

Error model (31), (32) shows that a constant mismatch between the wind speed measured at a distance in front of the turbine (and expected at the turbine site after some time) and wind speed measured at the turbine site can be well compensated via an integral part of the controller (28), which treats this mismatch as a constant disturbance. Resulting torque controller (28) is naturally composed of feedback and feedforward parts and represents a flexible and easy-to-upgrade structure, where the run-ahead model based feedforward part driven by the upwind speed measurements can easily be integrated into existing industrial feedback PI or PID turbine speed controller. Moreover, a constant or slowly varying tracking error offset due to mismatch between upwind and wind speeds can be well compensated via integral part of existing industrial controller.

Tracking performance of generator torque control (28) is illustrated in Figure 5 and Figure 6. Figure 5 shows tracking performance for different mismatch between upwind and wind speed measurements $\Delta V$. An expected desired turbine speed calculated via the upwind speed signal $V_{p}$ is plotted with a blue line, a desired turbine speed calculated via actual wind speed signal $V$ is plotted with a black line, and finally actual turbine speed regulated by controller (28) is plotted with a red line. The first subplot shows the case where $\Delta V=0$ (upwind and wind speeds are the same), the second subplot represents the case with a constant offset between upwind and wind speeds $\Delta V=$ const, and finally the third subplot shows the case of a stochastic normally distributed $\Delta V$.

Figure 6 shows the time chart of turbine speed tracking performance for normally distributed stochastic mismatch 
$\Delta V$ between the wind speed measured at a distance in front of the turbine (and expected at the turbine site after some time) and wind speed measured at the turbine site. The first subplot shows an expected and measured wind speeds at the turbine site. Expected wind speed is plotted with a blue line, and actual wind speed is plotted with a black line. The second subplot shows the tracking performance of the turbine speed. An expected desired turbine speed calculated via upwind speed signal $V_{p}$ is plotted with a blue line, a desired turbine speed calculated via actual wind speed signal $V$ is plotted with a black line, and finally actual turbine speed regulated by controller (28) is plotted with a red line. The third subplot shows a resulting generator torque calculated via (28), plotted with a black line with feedforward part driven by the upwind speed measurements plotted with a red line.

A satisfactory tracking performance is observed for zero, constant and stochastic offsets between the speed expected at the turbine and the actual speed.

Flapwise bending moment together with blade pitch regulation described in the next Section are shown in Figure 7, where a pitch angle regulation performance with the algorithm described in Section 4.2 is plotted in the first subplot. Desired pitch angle $\beta_{f}$ is plotted with a black line and actual pitch angle $\beta$ is plotted with a red line. Flapwise bending moment with its desired upper bound are plotted on the second subplot with black and red lines respectively.

Finally, Figure 8 shows that inclusion of the feedforward part to the controller improves tracking performance of the turbine speed control that in turn implies improvement of the power coefficient tracking performance and hence the turbine power production. Figure 8 shows the turbine speed control with and without feedforward part. Turbine speed that corresponds to control system with feedforward part is plotted with a red line on the first subplot. A blue line corresponds to control system without feedforward part. Desired turbine speed is plotted with a black line. Power coefficients as function of tip-speed ratio and blade pitch angle are plotted with the same lines on the second subplot. A desired power coefficient is plotted with a black line.

\subsection{Blade Pitch Angle Control and Load Regulation}

Pitch angle $\beta$ is associated with the control of mechanical loads of the turbine. The controller for pitch angle is based on look-up tables that are inverse to the flapwise bending moment look-up tables $M_{f}(\lambda, \beta)$ defined for a number of turbine speeds in Figure 2. Pitch angle associated with desired flapwise bending moment $M_{f d}$ is defined as $\beta_{f}(t)=M_{f}^{-1}\left(\lambda, M_{f d}\right)$, for the turbine speed $\omega_{r}$, regulated via control algorithm (28). As a desired value of the flapwise bending moment $M_{f d}$, its desired upper bound can be taken. In other words the flapwise bending moment is directly regulated to keep this moment bounded.

For a transient performance improvement the desired blade pitch angle as an input to pitch actuator (9) is defined as $\beta_{d}=\beta_{f}+\tau \dot{\beta}_{f}$, where $\dot{\beta}_{f}$ is an estimate of the derivative of $\beta_{f}$ calculated via a spline interpolation method mentioned in Section 3.1. Substituting $\beta_{d}$ in (9) yields the following exponentially stable closed loop
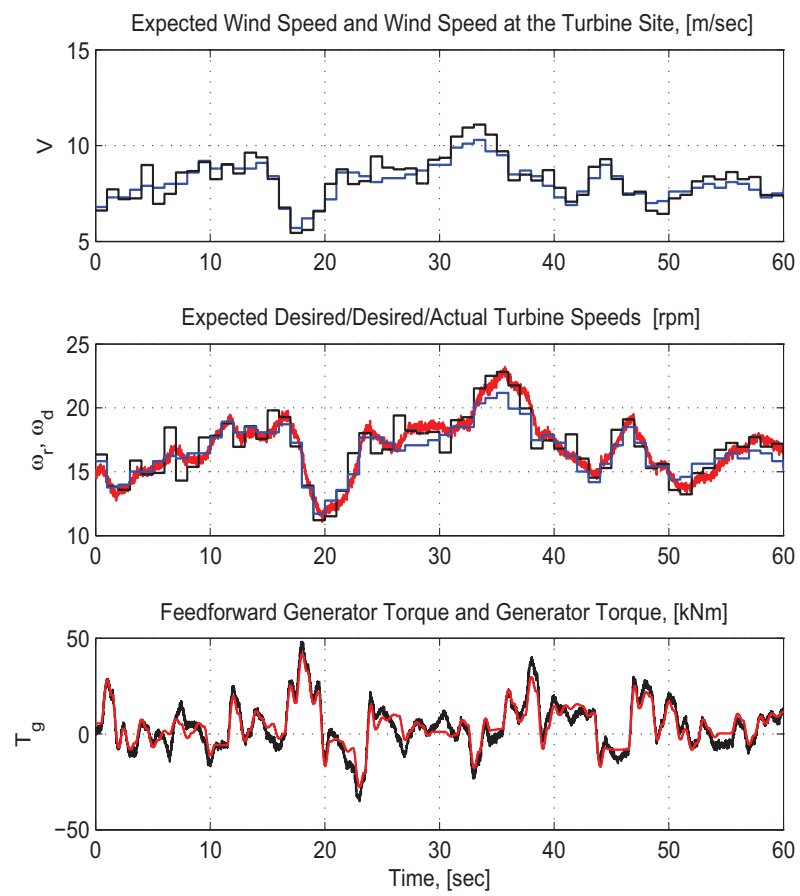

FIG 6. The time chart of turbine speed tracking performance for a normally distributed stochastic mismatch $\Delta V$ between wind speed measured at a distance in front of the turbine (and expected at the turbine site after some time) and wind speed measured at the turbine site.
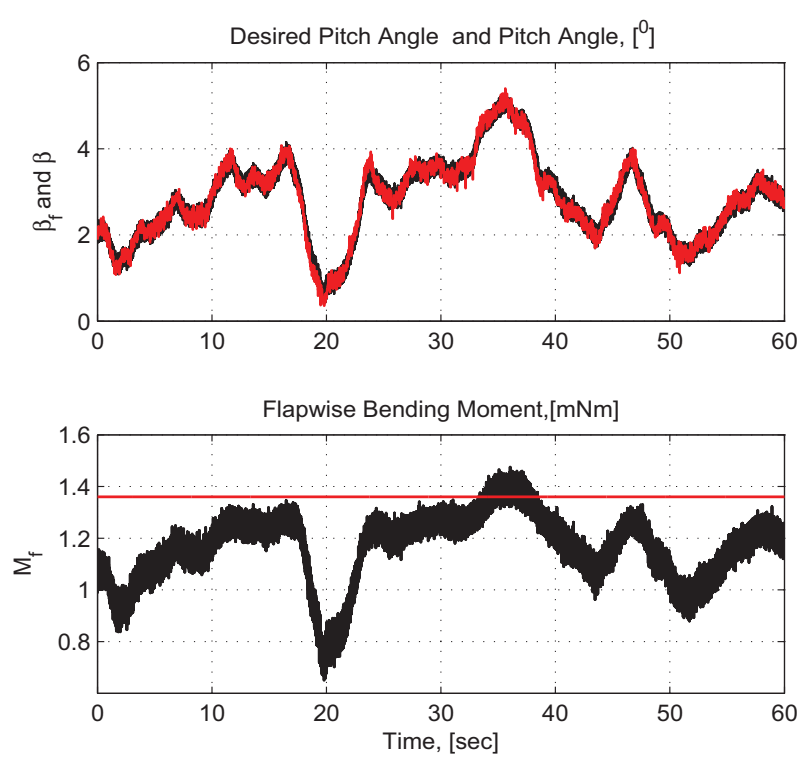

FIG 7. The time chart of pitch angle and flapwise bending moment in addition to Figure 6. 

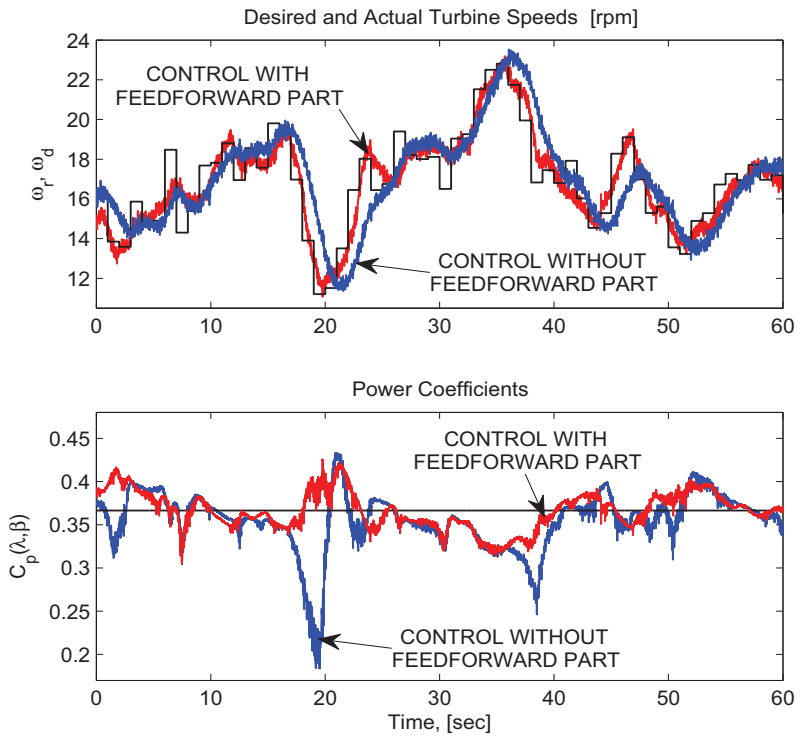

FIG 8. Comparison of tracking performance of two control systems: system with and without feedforward part.

dynamics $\dot{\beta}-\dot{\beta}_{f}=-\frac{1}{\tau}\left(\beta-\beta_{f}\right)$ when neglecting a communication delay. Usually a desired/command pitch angle $\beta_{f}$ is sent only to the pitch actuator $\left(\beta_{d}=\beta_{f}\right)$, which implies a slow response of the actuator. Introduction of the derivative term $\tau \dot{\beta}_{f}$ is equivalent to the prediction of the future state of command pitch angle $\beta_{f}$ with a simple predictor based on the first difference method: $\beta_{d}(t)=\beta_{f}(t)+\tau[\underbrace{\frac{\beta_{f}(t+\tau)-\beta_{f}(t)}{\tau}}_{\approx \dot{\beta}_{f}(t)}] \approx \beta_{f}(t+\tau)$. This prediction improves the transient performance of blade pitch actuation as it is illustrated in Figure 7.

\section{CONCLUSION}

A new concept of look-ahead modeling of the wind turbine response which results in feedforward part of the turbine controller is introduced. The concept creates an easy-to-upgrade control architecture where the run-ahead model based feedforward part driven by the upwind speed measurements can easily be integrated into an existing industrial feedback PI or PID turbine speed controller, driven by the wind speed measurements on the turbine site. Moreover, blade load regulation with improved performance can also be easily integrated into the proposed control architecture.

\section{ACKNOWLEDGEMENTS}

This work was supported by the Swedish Wind Power Technology Center (SWPTC). The authors are grateful to Magnus Ellsen and Ola Carlson from the SWPTC for providing wind speed measurements from the Hönö turbine outside Gothenburg, Sweden.

\section{REFERENCES}

[1] Kusiak A., Song Z., Zheng H., Anticipatory Control of Wind Turbines with Data-Driven Predictive Models, IEEE Transactions on Energy Conversion, vol. 24, No. 3, Sept. 2009, pp. 766-774.

[2] Bossanyi E., Savini B., Iribas M., Hau M, Fischer B., Schlipf D., van Engelen T., Rossetti M., and Carcangiu C., Advanced Controller Research for Multi-MW Wind Turbines in the UPWIND Project, Wind Energy, 2011, wileyonlinelibrary.com, DOI: $10.1002 /$ we.523.

[3] Johnson K., Pao L., Balas M., and Fingersh L, Control of Variable-Speed Wind Turbines: Standard and Adaptive Techniques for Maximizing Energy Capture, IEEE Control Systems Magazine, vol. 26, June 2006, pp. $70-81$.

[4] Henriksen L. and Poulsen N., Model Predictive Control of a Wind Turbine with Constraints, In Proceedings of EWEC 2008, Brussels, Belgium, March 2008.

[5] Laks J., Pao L., Wright A., Kelley N. and Jonkman B., The Use of Preview Wind Measurements for Blade Pitch Control, Mechatronics, 21, 2011, pp. 668-681.

[6] Pao L. and Johnson K., A Tutorial on the Dynamics and Control of Wind Turbines and Wind Farms, Proc. of American Control Conference, St. Louis, MO, USA June 10-12, 2009.

[7] Soltani M., Wisniewski R., Brath P. and Boyd S., Load Reduction of Wind Turbines Using Receding Horizon Control, IEEE Conference on Control Applications (CCA), Denver, CO, USA. September 28-30, 2011, pp. 852-857.

[8] Stotsky A. and Egardt B., Proactive Control of Wind Turbine with Blade Load Constraints, Proc. IMechE Part I: Journal of Sytems and Control Engineering, DOI: 10.1177/0959651812439976,online 27 March 2012.

[9] Wang N., Johnson K. and Wright A., LIDAR-based FX-RLS Feedforward Control for Wind Turbine Load Mitigation, American Control Conference, San Francisco, CA, USA June 29 - July 01, 2011, pp. 19101915.

[10] Sloth C., Esbensen T., Niss M., Stoustrup J., and Odgaard P., Robust LMI-Based Control of Wind Turbines with Parametric Uncertainties, Proc. of the 18th IEEE International Conference on Control Applications, Saint Petersburg, Russia, July 8-10, 2009, pp. 776-781.

[11] IEC 61400-1 Ed.3: Wind Turbines, Part 1: Design Requirements, International Electrotechnical Commission: Geneva, 2005-08.

[12] Ellsen M. and Carlson O., Drift, Utveckling och Dokumentation vid Chalmers Provstation för Vindenergiforskning, Chalmers Technical Report, Jan. 2009 (in Swedish).

[13] Burton T., Sharpe D., Jenkins N, and Bossanyi E., Wind Energy Handbook. Wiley, 2001.

[14] Stotsky A. and Forgo A., Recursive Spline Interpolation Method for Real-Time Engine Control Applications, Control Engineering Practice, vol. 12, 2004, pp. 409 - 416. 